\title{
Symbolic word clustering for medium-size corpora
}

\author{
Benoît Habert* and Elie Naulleau*** and Adeline Nazarenko* \\ *Equipe de Linguistique Informatique \\ Ecole Normale Supérieure de Fontenay-St Cloud \\ 31 av. Lombart, F-92260 Fontenay-aux-Roses \\ Firstname.Name@ens-fcl.fr \\ **Direction des Etudes et Recherches - Electricité de France \\ 1, av. du $\mathrm{G}^{a l}$ de Gaulle, F-92141 Clamart \\ Firstname.Name@der.edfgdf.fr
}

\begin{abstract}
When trying to identify essential concepts and relationships in a medium-size corpus, it is not always possible to rely on statistical methods, as the frequencies are too low. We present an alternative method, symbolic, based on the simplification of parse trees. We discuss the results on nominal phrases of two technical corpora, analyzed by two different robust parsers used for terminology updating in an industrial company. We compare our results with Hindle's scores of similarity.
\end{abstract}

Subjects Clustering, ontology development, robust parsing, knowledge acquisition from corpora, computational terminology

\section{Identifying word classes in medium-size corpora}

In companies with a wide range of activities, such as EDF, the French electricity company, the rapid evolution of technical domains, the huge amount of textual data involved, its variation in length and style imply building or updating numerous terminologies as NLP resources. In this context, terminology acquisition is defined as a twofold process. On one hand, a terminologist must identify the essential entities of the domain and their relationships, that is its ontology. On the other hand, (s)he must relate these entities and relationships to their linguistic realizations, so as to isolate the lexical entries to be considered as certified terms for the domain.

In this paper, we concentrate on the first issue. Automatic exploration of a sublanguage corpus constitutes a first step towards identifying the semantic classes and relationships which are relevant for this sublanguage.

In the past five years, important research on the automatic acquisition of word classes based on lex- ical distribution has been published (Church and Hanks, 1990; Hindle, 1990; Smadja, 1993; Grefenstette, 1994; Grishman and Sterling, 1994). Most of these approaches, however, need large or even very large corpora in order for word classes to bc discovered ${ }^{1}$ whereas it is often the case that the data to be processed are insufficient to provide reliable lexical information. In other words, it is not always possible to resort to statistical methods. On the other hand, medium size corpora (between 100,000 and 500,000 words: typically a reference manual) are already too complex and too long to rely on reading only, even with concordances. For this range of corpora, a pure symbolic approach, which recycles and simplifies analyses produced by robust parsers in order to classify words, offers a viable alternative to statistical methods. We present this approach in section 2. Section 3 describes the results on two technical corpora with two different robust parsers. Section 4 compares our results to Hindlc's ones (Hindle, 1990).

\section{Simplifying parse trees to classify words}

\subsection{The need for normalized syntactic contexts}

As Hindle's work proves it, among others (Grishman and Sterling, 1994; Grefenstette, 1994), the mere existence of robust syntactic parsers makes it possible to parse large corpora in order to automate the discovery of syntactic patterns in the spirit of Harris's distributional hypothesis. However, Harris' methodology implies also to simplify and transform each parse tree ${ }^{2}$, so as to obtain so-called "elementary sentences" exhibiting the main conceptual classes for the domain (Sager

\footnotetext{
${ }^{1}$ For instance, Hindle (Hindle, 1990) necds a six million word corpus in order to extract noun similarities from predicate-argument structures.

${ }^{2}$ Changing passive into active sentences, using a verb instead of a nominalization, and so on.
} 


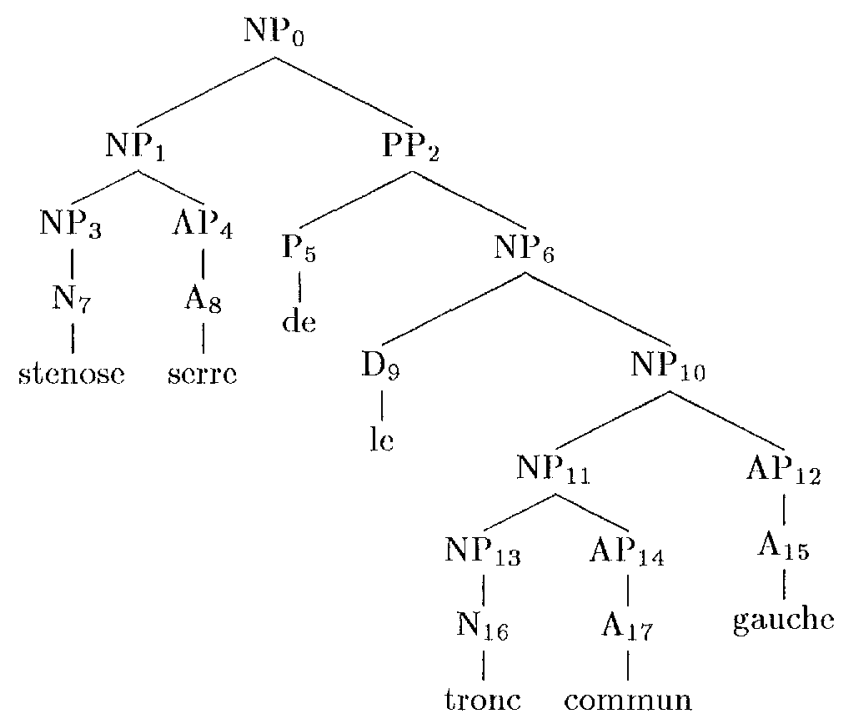

ligure 1: Parse tree for stenose serre de le trone commun gauche

et al., 1987).

In order to autorrate this normalization, we propose to post-process parse trees so as to emphasize the dependency relationships among tho content words and to infer semantic classes. Our approach can be opposed to the a prior onc which consists in building simplified representations while parsing (Basili et al., 1994; Metzler and Haas, 1989; Smeaton and Sheridan, 1991).

\subsection{Recycling the results of robust parsers}

For the sake of reusability, we chose to add a generic post-processing treatment to the results of robust parsers. It implies to transduce the trees resulting from different parsers to a common format.

Wo experimented so far two parsers: AlethGram and Lexter, which are being used at DERIDI for terminology acquisition and updating. 'They both analyze corpora of arbitrary length. AlethGram has been developped winthin the GRAAL project ${ }^{3}$. I.FXTTR has been developped at DER-EDF (13ourigault, 1993). In this experiment, we focussed on noun phrases, as they are central in most terminologies.

\subsection{The simplification algorithm}

The objective is then to reduce automatically the numerous and complex nominal phrases provided by AlethGram and LEXTER to elementary trees,

\footnotetext{
${ }^{3}$ The Eureka GR $\Lambda \Lambda \mathrm{I}$, project gathers in France GCI-ERI I (prime contractor), EDF, Aerospatiale and Renault.
}

which more readily exhibit the fundamental binary relations, and to classify words with respect to these simplified trees.

For instance, from the parse tree for stenose

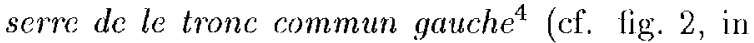
which non terminal nodes are indexed for reference purposes), the algorithm ${ }^{5}$ yields the set of elementary trees of figure 1 . 'The trees $a$ and $c$ correspond to contiguous words in the original sequence, whereas $b$ and $d$ only appear after modifier removal (sec below).

Two types of simplifications arc applicd when possible to a given tree:

1. Splitting: Each sub-tree irnmediately dominated by the root is extracted and possibly further simplified. For instance, removing node $\mathrm{NP}_{0}$ yields two sub-trees: $\mathrm{NP}_{1}$, which is elementary (see below) and $\mathrm{PP}_{2}$, which needs further simplification.

2. Modifier removal: Within the whole tree, every phrase which represents a modified constituent is replaced by the corrosponding non modified constituent. For example, in $\mathrm{Nl}_{0}$, the adjectival modifier serre is removed, as woll as the detcrminer and the adjectives

\footnotetext{
${ }^{4}$ Tight stcnosis of left common mainstem. In both parsers, the accents are removed during the analysis, the lemmas are used instead of inflected forms. Additionally, for simplification purposes, a contracted word like $d u$ is considered as a preposition-determiner sequence.

${ }^{5}$ See (Habert et al., 1995) for a detailled presentation. The corresponding software, SYCI,ADE, has been developped by the first author.
} 

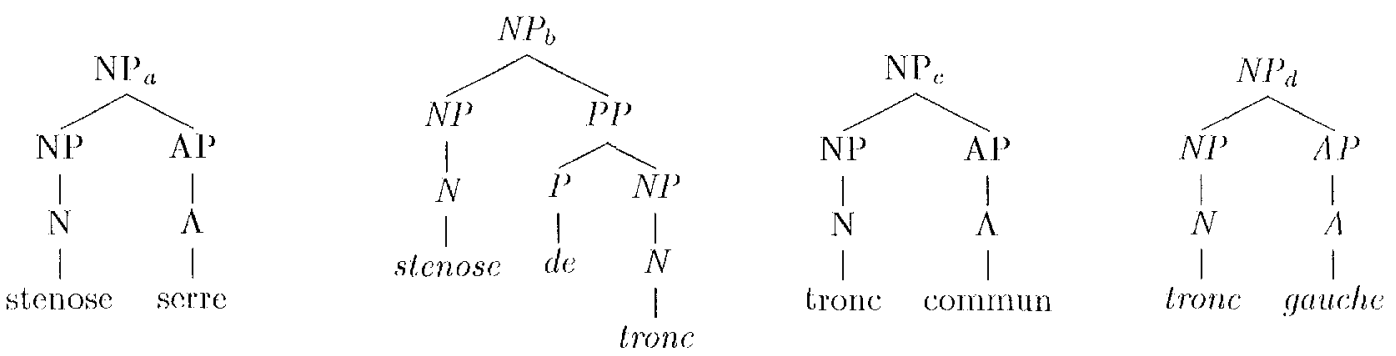

Figure 2: Whomontary trees for stenose serre de le trone commun gauche

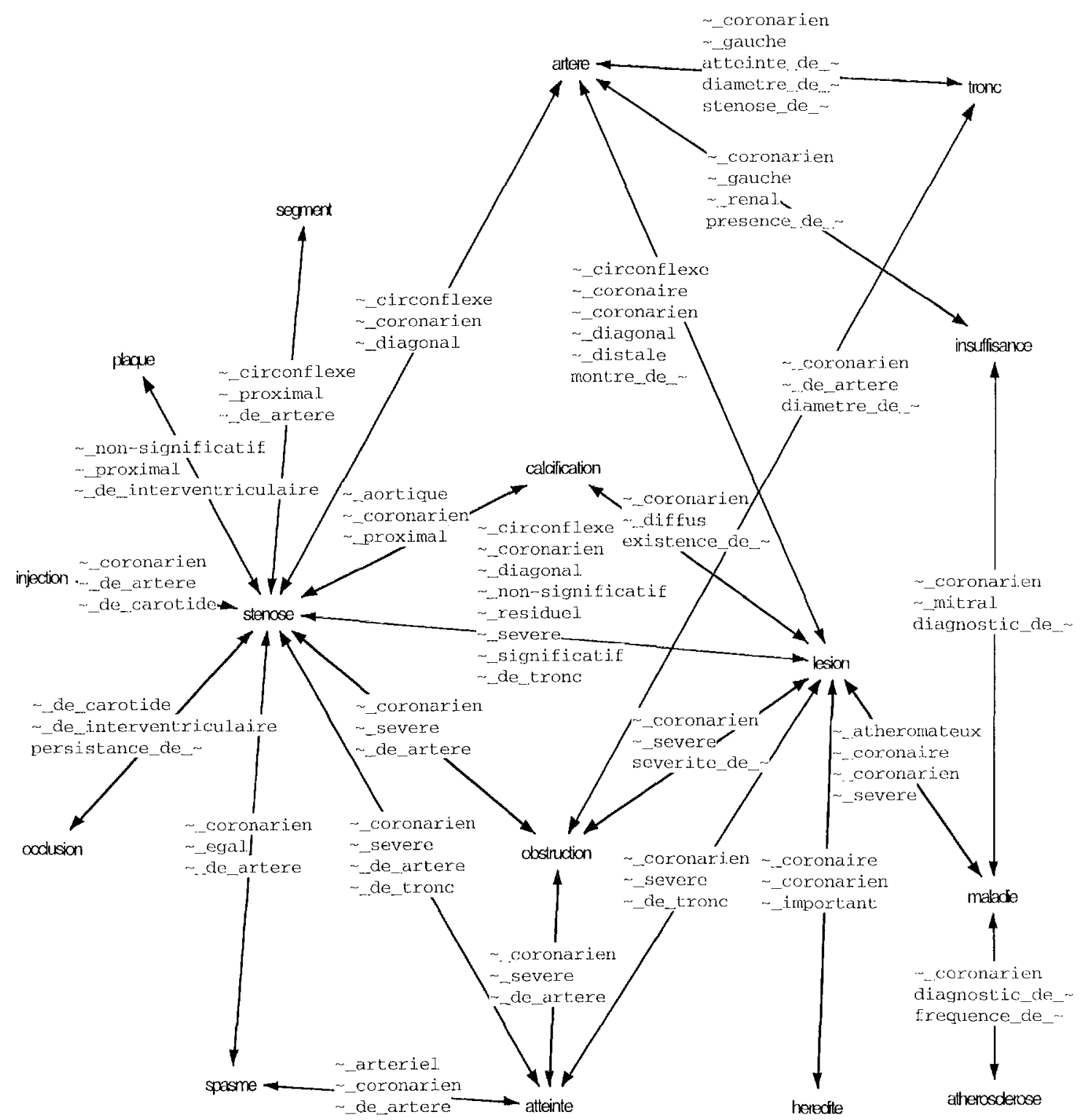

Figure 3: Example of a strongly connected component (CMC corpus) 
modilying trone, which loads to clementary tireo b.

When the corront troe is chementary, the simplification process stops. Before processing lihe set of original parse trees, one must dectare the trees which must not be simplified any further. In this experinent, are consiclered as elementary the nominal trees which exhibit, a binary relation bo. tween two "conteente" words, for instance between l.wo $\mathrm{N}$ in an $\mathrm{N}$ P' $\mathrm{N}$ secpuence.

\subsection{From elementary contexts to wored classes}

The resulting collocalions are coutrolled by the syntactic: relationships structuring the parse trees, which is not the case for window-hased approaches (chureh and llanks, 1990), even when they use part-of-spoech labols (Smadja, 1998; Daille, 1994). Tu the example, gauche is not related to stenose, as it. clocs not modify this noun.

'Tho elementary trees lead to sasses of syntactic contexts. lon instance, from the tree corresponding to stenose serre, two classes of contexts are created. 'l'te lirst one, slenose $\sim$, in which $\sim$ stands for the pivot, word, contains seres, whereas 1. he second one, $\sim$ serre, contains stenose At the end of the simplitication peocess, hese chasses have been completed and oblier ones areated. Wo chim that the semantic similarily between two lexical ontries is in proportion with the number of shared contexts. For instance, in one of our corpora, stenose shares 8 contexts with lesion.

In order ho get a global vision of the sitrilaritiss relying on elementary contexts, a griph is computed. 'The words constitute the nodes. A link corresponds to a certain number of shared contexts (according to a chosen lareshold). 'The edges are labelled with the shared contexts. The strongly connected components ${ }^{6}$ and the elicpues" are computed as woll, as they are the most relevant parts of the graph, on topological groumds. 'lle underlying intution is that a connected contponent relates neighboring words (Bensch and Savitch, 1995) and that the cliques teurl to isolato similarity classes. $A_{n}$ extract of a connected component, with 3 as a threshold, appoars in ligure. 3.

\footnotetext{
${ }^{6}$ The sub-graples in which there is a path between every pair of distinct nodes.

7'flhe sub-graptes in which there is a path between cach node and enery other node of the graph.
}

\section{Results}

\subsection{Two corpora}

We have tested our method on two technical medium-size corpora. The first one, the Now clear 'Technology (corpus (N'TC) of lidel', is of about 52,000 words. 'The second one, he core nary Medicine (orpus (CM(), is of about 60,000

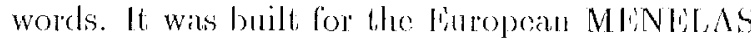
project (\%weigenbantm, 1994) and is used lor pilot studies in terminology extraction".

\subsection{A visual map of concepts and relationships}

fiven if no ontology an be fully automatically derived from a corpus (Ilabert and Natyarenlio, 1996), the $\$ Y(1, \Lambda I)$ lig graphs can loe lised lo bootstrap the building of the ontology of a domain.

'lla: SYCIAD): network gives a glotal viow over the conpus which enables an alternatio paradigmatic and syntigmatic explomation of l.he context of a word. 'I'te graph enables to identily the concepts, their possible typical properties, and also the selationships between the selected conceptss.

The clicues bring ont small paradigmatice sets of forms which, in a first step, can be intor preted ats ontological chasses reflecting conceptis. 'l'he are labels then help lo reline those classess by ardeling some of the surromeling words which are not part of the cligue but which acverthe less share the most siguilicant or some similar contexts. From the clicue fstenose, lesen, ofstruction, alleinte) (ce. lig. 3), one can build tho chass of alfections which are located in the body as \{plaque, occlusion, stenose, lesion, calcificalion, obstrution, ablemte?. Similarly, from the graph of the (MMC eorpus, one can identily the classes of borly sites \{atere, branche, resean, ventricule, interventriculaire, corotide\}, of cliseases \{maladie, artherosclerose $\}$ and of chirurgical acts $\{$ pontage, rovascularisation, angioplastir? .

Once these concepts are identified, their properties ean be listed, by interpreting the labels of the links. 'The attribute of the localization of the allicetions is described through three kinds of modiliers (fig. 3): bouns ( $\sim$ de $\{$ artere, trone $\}$ ), names of arteries ( $\sim$ de $\{c a m b t i d e$, inlerventivilaire $\}$ and adjectives related to a specific artery $(\sim$ fromoname, coronarion, diagonal, cinconflexe $\}$ ). The attibutio degree of the affection is also revealed through $\{\sim$ significatif, non-significatif, scvere, important, severile\}.

\footnotetext{
${ }^{8}$ Aroupe Terminologic et Intelligence Artificielle PRC.(1)R Intelligence Artificicllo, CNRS
} 


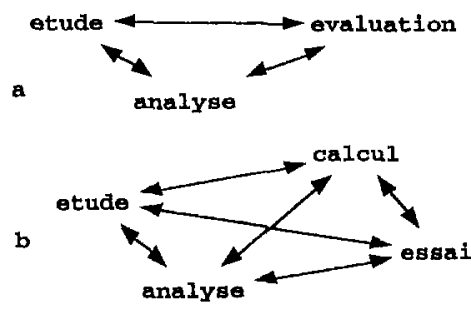

Figure 4: Polysemy of etude

Last, relationships between concepts can be extracted, such as the "part-of" relation between tronc and artere, and segment and artere (fig. 3).

\subsection{Distinguishing word meanings}

Polysemy and quasi-synonymy often makes the ontological reading of linguistic data difficult. However, through cliques and edge labels, the SYCLADE structured and documented map of the words helps to capture the word meaning level.

Among a set of connected words where $w$ is similar to $w_{i}$ and $w_{j}$, cliques bring out coherent subsets where $w_{i}$ and $w_{j}$ are also similar to each other. We argue that the various cliques in which a word appears represent different axes of similarity and help to identify the different senses of that word. For instance, in the whole set of words connected to etude (study) in a strongly connected component of the NTC graph (analyse, evaluation, resultat, presentation, principe, calcul, travail...), some subsets form cliques with etude. Two of those cliques (resp. $a$ and $b$ in fig. 4 - threshold of 7) bring out a concrete and a more theoretical use of etude.

The network also enables to distinguish the uses of quasi-synonyms such as coronaire and coronarien in the CMC corpus. Even if they are among the most similar adjectives ( 7 shared contexts) and if they belong to the same clique \{coronaire, coronarien, diagonal, circonflexe $\}$, the fact that coronarien alone is connected to evaluation adjectives (severe, significatif and important) shows that they cannot always substitute to each other.

\section{Towards an adequate similarity estimatation for the building of ontologies}

The comparison with the similarity score of (Hindle, 1990) shows that SYCLADE similarity indicator is specifically relevant for ontology bootstrap and tuning. Hindle uses the observed frequencies within a specific syntactic pattern (sub- ject/verb, and verb/object) to derive a cooccurrence score which is an estimate of mutual information (Church and Hanks, 1990). We adapted this score to noun phrase patterns. ${ }^{9}$ However the similarity measures based on cooccurrence scores and nominal phrase patterns are less relevant for an ontological analysis. The subgraph of the chirurgical acts words, which is easy to identify from the SYCLADE graph (fig. 5a), is split in different parts in the similarity graph (fig. 5b). This difference stems from the fact that this cooccurrence score overestimates rare events and underlines the collocations specific to each form..$^{10}$ For instance, it appears that the relationship between stenose and lesion, which was central in figure 3 , with 8 shared contexts, almost diseappears if one considers the number of shared cooccurrences. Therefore, similarity measures based on cooccurrences and similarity estimation based on shared contexts must not be used in place of each other.

As opposed to Hindle's lists of similar words which are centered on pivot words whose neighbors are all on the same level, in SYCLADE graphs, a word is represented by its role in a whole syntactic and conceptual network. The graph enables to distinguish the various meanings of words, a crucial feature in the ontological perspective since the meaning level is closer to the concept level than the word level. In addition, the results are clear and more easily interpretable than those given by a statistical method, because the reader does not have to supply the explanation as to why and how the words are similar.

The building of an ontology, which is a timeconsuming task and which cannot be achieved automatically, can nevertheless be guided. 'The SYCLADE graphs based on shared contexts can facilitate this process.

${ }^{9}$ For instance, for $N_{1} P N_{2}$

$$
\operatorname{CoOc}_{N_{1} P N_{2}}=\log 2 \frac{\frac{f\left(N_{1} P N_{2}\right)}{k}}{\frac{f\left(N_{1}\right)}{k} \cdot \frac{f\left(N_{2}\right)}{k}}
$$

where $f\left(N_{1} P N_{2}\right)$ is the frequency of noun $N_{1}$ occurring with $N_{2}$ in a noun preposition pattern, $f\left(N_{1}\right)$ is the frequency of $N_{1}$ as head of any $N_{1} P N_{x}$ sequence and $f\left(N_{2}\right)$ the frequency of $N_{2}$ in modifier/argument position of any $N_{x} P N_{2}$ sequence and $k$ is the count of $N_{x} P N_{y}$ elementary trees in the corpus. Cooc $N A d_{j}$ and $C_{o o c} \operatorname{Adj}_{N}$ are similarly defined.

${ }^{10}$ The various cooccurrence scores retrieve sets of collocations which are sharply different from the contexts shown by SYCLADE connected components. The collocations which get the greatest cooccurrence scores seem to characterize medecine phraseology ( $f a c$ teur (de) risque, milieu hospitalier) but not the coronary diseases as such. 

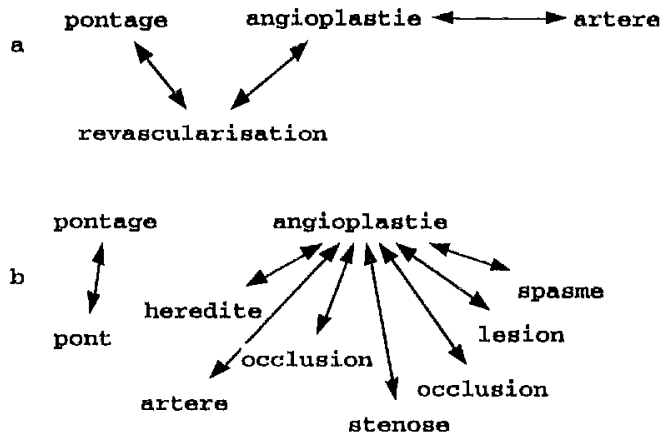

ligure 5: Similarity among the chirurgical act family

\section{Acknowledgments}

We thank Christian Jacquemin (IRIN), Didier Bourigault, Marie-Luce Herviou, JeanDavid Sta (DER F,DF), Marie-Hélène Candito (TALAN for their remarks on a previous version of this paper. We are very grateful to Serge Heiden (1BLI), who has devcloped (araphX (ftp://mycroft.ens-fcl.fr/pub/graph $/$ ), the graph interactive handling software that enabled us to visualize and handle the SYCLADE: graphs.

\section{References}

Roberto Basili, Maria-Teresa Pazienza, and Paola Volardi. 1994. A "not-so-shallow" parser for collocational analysis. In Proceedings of Coling'94, pages 447-453.

Peter $\Lambda$. Bensch and Walter J. Savitch. 1995. An occurrencc-based model of word categorization. Annuals of Mathemalics and Artificial Intelligence, $11: 1-16$.

Didier Bourigault. 1993. An endogenous corpusbased method for structural noun phrase disambiguation. In 6th Luropean Chapter of the Association for Computational Linguistics.

Kenneth W. Church and Patrick Ilanks. 1990. Word association norms, mutual information, and lexicography. Computational linguistics, 16(1):22-29, march.

Bćatrice Daille. 1994. Approche mixte pour l'cxtraction de terminologie : statistique lexicale el filtres linguistiques. PhD Thosis, Paris VII University, february. Supervisor: Isaurence Danlos.
Gregory Grefenstette. 1994. Exploration in Automatic Thesaurus Discovery. Kluwer Academic Publishers.

Ralph Grishman and John Sterling. 1994. Generalizing automatyically generated selectional patterns. In Proceedings of Coling'94, volume 3 , pages $742-747$, Kyoto.

Benoît Habert and Adeline Nazarenko. $1996^{\circ}$ La syntaxe comme parche-pied de l'acquisition des connaissances. In Actes des Journées d'Acquisition des Connaissances, Sète, May.

Benoît Habert, Philippe Barbaud, J'ernande Dupuis, and Christian Jacquemin. 1995. Simplifier des arbres d'analyse pour dégager. les comportements syntaxico-sémantiques des formes d'un corpus. Cahiers de Grammaire, $(20)$.

Ionnald Hindle. 1990. Noun classification from predicate-argument structures. In l'roceedings of the Association for Computational linguistics', pages 268-275.

Douglas P. Metzler and Stephanie W. Haas. 1989. The constituent object parser : Syntactic structure matching for information retrieval. In Procecdings, loth Annual International ACM STGIR Conference on Research and Developmenl in Information Retrieval (SIGIR'89), pages 117-126, Cambridge, MA.

Naomi Sager, Carol Friedman, and Margared S. I.yman (editors). 1987. Medical language Processing: Computer Management of Narrative Data. Addison-Wesley.

Pranck Smadja. 1993. Retrieving collocations from text: Xtract. Computational Iinguistics, 19(1):143-177, march. Special Issue on Using Large Corpora: I.

Alan Smeaton and P. Sheridan. 1991. Using morpho-syntactic language analysis in phrase matching. In Proceedings RIAO'91, pages 415 429.

Pierre Zweigenbaum. 1994. Menelas: an access system for medical records using natural language. Computer Methods and Programs in Biomedicine, 15:117-120. 